\title{
IMPORTÂNCIA NA REGULAÇÃO DA TEMPERATURA CORPORAL APLICADA À PRÁTICA DE ATIVIDADE FÍSICA
}

\author{
Wagner Pires Camargo ${ }^{1}$ \\ Ana Carolina Gonçalves Pontes ${ }^{2}$ \\ Ingrid Barreto Alves ${ }^{3}$ \\ Dr $^{\text {a }}$ Ana Cristina de Sousa Murray ${ }^{4}$
}

\section{RESUMO}

Termorregulação e seus aspectos e mecanismos, físicos, fisiológicos e patológicos associados à prática de atividades físicas. Os mecanismos de defesa do corpo estão em constante trabalho para manter normotermia, utilizando sensores localizados na pele para cada tipo de situação; através de vias aferentes e eferentes, o hipotálamo trabalha para manter sempre à homeostasia na temperatura central, na tentativa de evitar a desregulação que pode ocasionar a morte. Devemos tomar cuidados necessários, como, vestimentas adequadas para a prática de atividades físicas, evitar praticar atividades em temperaturas extremas, além de outros fatores que podem ocasionar hipertermia ou hipotermia, levando à necrose celular e/ ou a morte.

Palavras chave - Crianças, Jovens, Termorregulação, Fisiologia, Exercício Físico, Mecanorreceptores.
\end{abstract}

\section{INTRODUÇÃo}

A regulação de temperatura corporal central é critica por que as estruturas celulares e as vias metabólicas são afetadas pela temperatura. Por exemplo, as enzimas que regulam as vias metabólicas são muito influenciadas pelas alterações de temperatura. Um aumento da temperatura corporal acima de $45^{\circ} \mathrm{C}$ (a temperatura normal é de aproximadamente $37^{\circ} \mathrm{C}$ ) pode destruir a estrutura protéica das enzimas, resultando em morte, enquanto a diminuição abaixo de $34^{\circ} \mathrm{C}$ pode causar uma lentidão do metabolismo e uma função cardíaca anormal (arritmias). Por essa razão, os seres humanos e muitos animais passam toda a vida a apenas alguns graus de seu ponto de morte pelo calor. Portanto a temperatura corporal deve ser cuidadosamente regulada. (Scott K. Powers., Edward T. Howley). Esse descritivo tem como objetivo abordar os mecanismos, físicos, fisiológicos e patológicos da desregulação na temperatura central e cutânea e a importância na homeostasia da temperatura corporal.

\section{METODOLOGIA}

Pesquisa base de dados, Artigos científicos e Revisão bibliográfica. Termorregulação

\footnotetext{
${ }^{1}$ Formando do curso de Licenciatura em Educação Fisica da Faculdade de São Paulo - Centro Novo Formando do curso de Licenciatura em Educação Fisica da Faculdade de São Paulo - Centro Novo Formando do curso de Licenciatura em Educação Fisica da Faculdade de São Paulo - Centro Novo ${ }^{4}$ Doutoranda do curso de Fisioterapia FOFITO/USP e Mestre em Reabilitação pela UNIFESP/EPM, revisora cientifica do artigo.
}

Revista Acadêmica Multidisciplinar da Faculdade Patos de Minas 
A manutenção da temperatura corporal constante exige que a perda de calor esteja coordenada com a taxa de produção de calor e para obter se a regulação térmica, o corpo humano possui mecanismos nervosos e hormonais que regulam tanto a taxa metabólica quanto a quantidade da perda de calor em resposta ás alterações (Scott K. Powers., Edward T. Howley).

O ser humano por ser homeotérmico, isto é, possui a capacidade de manter a temperatura corporal dentro de um intervalo pré-determinado apesar das variações térmicas do meio ambiente (homeostasia térmica). (Magalhães, Sónia et al).

A variação térmica circadiana é um fenómeno natural e geralmente não ultrapassa os $0.6^{\circ} \mathrm{C}\left(1^{\circ} \mathrm{F}\right)$. A temperatura corporal é menor pela manhã, aumenta ao longo do dia e é máxima pelo início da noite. (Magalhães, Sónia et al.)

0 equilíbrio térmico é conseguido através do balanço entre a perda e a produção ou aquisição de calor. Seguidamente serão abordados os mecanismos físicos e fisiológicos que contribuem para este equilíbrio. (Magalhães, Sónia et al.)

\section{CONCEITO DE TERMOGÉNESE}

A termogénese corresponde à energia na forma de calor gerada ao nível dos tecidos vivos. A quantidade de calor produzida é diretamente proporcional à taxa de metabolismo corporal (40-60\% da energia proveniente da hidrólise do trifosfato de adenosina - ATP, é perdido sob a forma de calor). (Magalhães, Sónia et al.)

A taxa de metabolismo corporal depende dos seguintes fatores:

- Taxa de metabolismo basal de todas as células corporais (para cada aumento da temperatura no valor de $1{ }^{\circ} \mathrm{F}$ ou $0.6^{\circ} \mathrm{C}$, esta taxa aumenta aproximadamente $10 \%$ );

- Taxa de metabolismo adicional decorrente da atividade muscular;

- Taxa de metabolismo adicional secundário ao efeito da Tiroxina (e em menor grau por outras hormonas como a hormona de crescimento ou a testosterona) a nível celular:

- Taxa de metabolismo adicional causada pelo efeito da epinefrina, norepinefrina e pela estimulação simpática a nível celular;

- Taxa de metabolismo adicional por um aumento intrínseco da atividade química nas próprias células.

A contribuição de cada fator para o metabolismo varia ao longo do tempo. Por exemplo numa situação de repouso a termogênese é decorrente essencialmente do metabolismo basal. Enquanto na prática de exercício físico deriva principalmente da atividade muscular.

Produção e perda de calor

Como já vimos acima nesse descritivo o objetivo de regulação de temperatura é a manutenção da temperatura central constante. Essa regulação é 
conseguida por meio do controle da taxa de produção e de perda de calor. Quando em desequilíbrio, o corpo ganha ou perde calor. 0 centro de controle da temperatura é uma área no cérebro denominado hipotálamo. Ele funciona como um termostato, indicando o aumento da produção de calor quando a temperatura corporal cai e aumentando a taxa de perda de calor quando a temperatura aumenta. A regulação da temperatura é controlada tanto por processos químicos quanto físicos. (Scott $\mathrm{K}$. Powers., Edward T. Howley).

\section{MECANISMOS FÍSICOS DE TRANSFERÊNCIA DE CALOR}

A energia térmica pode ser absorvida a partir do meio externo ou dissipada para o mesmo (conforme o gradiente térmico). Os principais mecanismos implicados são a radiação, a condução e a convecção. (Magalhães, Sónia et al.)

Radiação

Se a temperatura do corpo de um ser humano for superior à temperatura do meio externo, uma maior quantidade de calor irá irradiar a partir do corpo do que a que irá ser irradiada para o corpo, isto é, ocorre perda de calor por parte do organismo. (Magalhães, Sónia etal.)

Condução

A condução é um mecanismo de transferência direta de calor. 0 calor é a energia cinética do movimento molecular e pode ser transferido de umas moléculas para outras, é neste processo que consiste a condução. (Magalhães, Sónia et al.)

Taxa de metabolismo basal: quantidade de calor produzida no estado de repouso em presença de um ambiente térmico neutro onde nenhuma transferência de calor ocorre entre o organismo e o meio ambiente. (Magalhães, Sónia et al.)

\section{Convecção}

Pelo que foi descrito acima, percebe-se que a condução é um mecanismo auto-limitado (existe apenas até ao momento em que as moléculas possuam uma energia cinética equivalente), contudo se o ar adjacente ao corpo for removido e substituído por um ar "novo", o equilíbrio jamais será atingido - a transferência de calor pelo meio de correntes de ar é chamada de convecção. (Magalhães, Sónia et al.)

Quanto maior a velocidade das correntes de ar (maior renovação do ar adjacente ao corpo), maior a amplitude da transferência de calor. (Magalhães, Sónia et al.)

Este processo é semelhante na situação de o organismo estar submergido em água, com a diferença que a água possui uma maior capacidade de absorção e condução para o calor (perda de calor muito mais rápida). (Magalhães, Sónia etal.)

0 vestuário minimiza as perdas de calor por condução e convecção ao permitir a criação de uma camada de ar, não renovada, junto à superfície corporal. 
Contudo, esta capacidade perde-se quando as roupas se tornam molhadas ou húmidas (por exemplo em roupa suada), devido à elevada condutibilidade da água que aumenta a taxa de transferência de calor através da roupa em 20 vezes ou mais. (Magalhães, Sónia et al.)

Em situações usuais, os mecanismos físicos acima citados promovem perda de calor pelo corpo, quando a temperatura ambiente é inferior à temperatura corporal. (Magalhães, Sónia et al.)

Contudo em situações em que a temperatura ambiente seja superior a $37^{\circ} \mathrm{C}$, por exemplo, em uma sauna (temperatura ambiente $\sim 70{ }^{\circ} \mathrm{C}$ ) verifica-se uma inversão destes mecanismos com ganho de calor pelo organismo através dos mesmos processos físicos.

\section{MECANISMOS FISIOLÓGICOS DE TRANSFERÊNCIA DE CALOR}

\section{Controle Vasomotor}

\section{Transmissão de Calor Corporal Central para a Pele}

A pele e as extremidades, contrariamente ao que acontece ao nível das regiões corporais mais profundas (região corporal central), têm maior variação de amplitude térmica. (Magalhães, Sónia et al.)

0 tecido celular adiposo tem função isolante natural (baixa condução de calor), e separa a pele (região mais sensível às variações térmicas externas) da região corporal central (temperatura mais estável). (Magalhães, Sónia et al.)

O fluxo sanguíneo cutâneo estabelece ligação entre a pele e a região corporal central. (Magalhães, Sónia et al.)

A irrigação cutânea é composta por um sistema complexo de ramificações vasculares, do qual fazem parte plexos venosos, arteríolas e anastomoses arteriovenosas (essencialmente presentes ao nível de áreas expostas como os pés, as mãos, o nariz e os pavilhões auriculares). 0 plexo venoso subcutâneo é abastecido pelas arteríolas e anastomoses arteriovenosas, de forma que o fluxo de sangue subcutâneo irá variar conforme o maior ou menor grau de vasoconstrição destas últimas. A modulação do tónus arterial depende preponderantemente do sistema nervoso simpático. (Magalhães, Sónia etal.)

Dessa forma, o maior ou menor aporte sanguíneo medeia o fluxo de calor interno para a pele, a partir da qual o calor pode ser posteriormente dissipado para o meio ambiente. A condução de calor ao passar de um estado de vasoconstrição total para vasodilatação total aumenta cerca de 8 vezes. Poderá então se afirmar que a pele funciona como um sistema de radiação de calor controlado. (Magalhães, Sónia et al.) 


\section{REGULAÇÃO DA TEMPERATURA CORPORAL}

Para a manutenção de uma temperatura corporal estável, é essencial a integridade de todos os elementos envolvidos na sua regulação, nomeadamente os sensores térmicos, o centro integrador e de comando e os sistemas eferentes. (Magalhães, Sónia et al.)

\section{SENSORES TÉRMICOS}

\section{Hipotálamo Anterior e Área Pré-óptica}

Contêm neurónios sensíveis ao calor e neurónios sensíveis ao frio e são estimulados por variações da temperatura do sangue que perfunde essa área - rede vascular especializada com função de barreira hematoencefálica limitada denominada organum vasculosum laminae terminalis. A estimulação térmica destes neurónios traduz-se por um aumento da frequência dos impulsos emitidos por segundo. (Magalhães, Sónia et al.)

\section{Receptores cutâneos térmicos}

São de dois tipos: sensíveis ao frio, (receptores de Krause) ou sensíveis ao calor, (receptores de Ruffini). A informação transmitida por estes receptores é enriquecida pela informação proveniente de receptores da dor, especificamente estimulados por variações extremas da temperatura, o que explica que estas possam ser percebidas como dor, ou seja no caso de um estimulo mecânico excessivo de frio ou calor, entra em ação as terminações nervosas livres, receptores sensíveis aos estímulos mecânicos térmicos e especialmente aos dolorosos, inativando os receptores de Krause e de Ruffini.

Os receptores térmicos localizam-se imediatamente abaixo da pele e distribuem-se em diferentes percentagens consoante a área corporal (por exemplo, no caso dos receptores do frio - nos lábios $15-25 / \mathrm{cm} 2$, nos dedos $3-5 / \mathrm{cm} 2$, no tórax $1 / \mathrm{cm}^{2}$ ). Os receptores do frio são consistentemente mais numerosos, contudo a relação entre receptores frio/calor pode variar de 3:1 a 10:1. A existência de um maior número de receptores sensíveis ao frio deve-se ao facto de, num meio ambiente neutro, a taxa metabólica do ser humano produzir consistentemente mais calor do que é necessário para manter a temperatura corporal central a 37ํㅡ. (Magalhães, Sónia etal.)

A informação dos receptores térmicos progride juntamente com a informação dos receptores dolorosos cutâneos no interior de fibras $C$ não 
mielinizadas (velocidade de transmissão $0.4-2 \mathrm{~m} / \mathrm{s}$ ), e de fibras A delta pequenas mielinizadas (velocidade de transmissão $20 \mathrm{~m} / \mathrm{s}$ ) até à lamina superficial do corno dorsal da medula espinal. Seguidamente cruzam a linha média, dirigindo-se então no sentido ascendente através do trato espinotalâmico contralateral até à formação reticular pontina e os núcleos posterolateral ou ventrolateral do tálamo. A informação progride posteriormente para o hipotálamo. (Magalhães, Sónia et al.)

\section{CENTRO INTEGRADOR}

Os sinais provenientes de todos os tipos de receptores citados anteriormente são integrados ao nível do hipotálamo $\rightarrow$ centro integrador.

Após a integração das diferentes informações aferentes e comparação das mesmas com o ponto de regulação térmica, são emitidas informações para diversos orgãos ou sistemas eferentes dependendo do tipo de resposta a estimular promoção do ganho ou da perda de calor. (Magalhães, Sónia et al.)

\section{SISTEMAS EFERENTES}

\section{Sistema Nervoso Central}

Ao nível do sistema nervoso central, mais propriamente no córtex cerebral, a percepção de variações da temperatura leva a alterações comportamentais, isto é, respostas voluntárias, importantes na prevenção da hipo ou hipertermia. Incluem o deslocamento para áreas mais quentes ou mais frias, remoção ou adição de roupas, diminuição ou aumento da atividade, e aumento ou diminuição das áreas de pele exposta. (Magalhães, Sónia et al.)

\section{Sistema Nervoso Autónomo}

Tónus vascular (vasoconstrição vs. vasodilatação) - Mecanismo Cutâneo de Radiação. (Magalhães, Sónia etal.)

Sudorese e frequência respiratória (quanto mais elevada, maiores serão as perdas insensíveis através dos pulmões; é um mecanismo de perda de calor pouco ativo no ser humano contrariamente ao que ocorre noutros animais) - Mecanismo de Evaporação. (Magalhães, Sónia etal.)

Metabolismo celular. 0 metabolismo celular pode ser uma forma de termogénese química, e consiste na produção de energia sob a forma de calor através da fosforilação oxidativa eficiente ou ineficiente (isto é, que não leva a formação de ATP sendo que toda a energia é libertada sob a forma de calor) de nutrientes intracelulares. (Magalhães, Sónia et al.)

Lipólise da gordura castanha (gordura termogénica). A gordura castanha pode ser 
considerada uma fonte de termogénese química dada a existência no interior deste tipo de adipócitos de mitocôndrias especializadas na oxidação ineficiente (isto é, que não leva a formação de ATP). Nos recém-nascidos, onde ela existe em quantidade considerável (essencialmente ao nível do espaço inter-escapular), é a fonte principal de calor. Nos adultos, dado existir em escassa quantidade (principalmente à volta dos orgãos internos e Aorta), contribui somente para 10-15\% da quantidade de calor produzida. (Magalhães, Sónia etal.)

Piloerecção. É um importante mecanismo de preservação de calor nos animais e consiste na contração do músculo eretor do pêlo presente nos folículos pilosos. A contração em bloco daqueles leva à ereção conjunta dos pêlos retendo junto à pele uma camada de ar mais ou menos constante (camada isolante), o que permite uma menor perda de calor para o meio externo - Mecanismo inibidor da condução e convecção. (Magalhães, Sónia et al.)

\section{Sistema Nervoso Somático}

Comanda a contração muscular (fonte importante de energia térmica termogénese muscular). Pode ser estimulado pelo córtex cerebral ou pode ser estimulado involuntariamente pelo hipotálamo.

Ao nível do hipotálamo posterior existe um centro motor primário que modula o grau de inibição da atividade dos neurónios motores anteriores presentes na medula espinal. A diminuição da inibição dos neurónios anteriores (promovida por diminuição da temperatura corporal central abaixo do valor de regulação) leva numa fase inicial ao aumento do tónus muscular e posteriormente, se mantida, ocorrem contrações repetitivas, isto é, tremores. A contração rápida involuntária da musculatura esquelética pode resultar num aumento de 4 vezes da produção de calor , de 2 vezes do consumo de oxigénio e de 6 vezes da taxa metabólica. (Magalhães, Sónia etal.)

\section{Hipófise}

Termogénese química: pode provir da lipólise da gordura castanha ou branca, da glicogenólise, principalmente à nível muscular e hepático, ou da hidrólise do ATP. (Magalhães, Sónia et al.)

O hipotálamo tem capacidade de estimular determinadas substâncias que funcionam como hormonas, uma das quais é chamada de hormona neurosecretora libertadora de Tirotrofina. Esta última é libertada para as veias portais hipotalámicas sendo transportada até à hipófise onde promove a libertação da hormona libertadora da Tiroxina (TSH). A TSH, por sua vez, ao passar para a corrente sanguínea leva à libertação de Tirotoxina (T4) pela tireóide. (Magalhães, Sonia et al.) 
A Tirotoxina estimula o metabolismo celular, pelo que se percebe que uma diminuição ou aumento da sua produção origina, respectivamente, um aumento ou diminuição da energia térmica produzida. (Magalhães, Sónia et al.)

\section{Termostato de corpo - Hipotálamo}

Enfatizando, o centro termorregulador do corpo está localizado no hipotálamo. 0 hipotálamo anterior é responsável, sobretudo, por controlar o aumento do calor corporal, enquanto o hipotálamo posterior é responsável, pela reação a uma diminuição da temperatura corporal. Em geral, o hipotálamo funciona de modo similar a um termostato doméstico, isto é, ele tenta manter uma temperatura central constante em torno do "ponto de ajuste". A temperatura do ponto de ajuste dos seres humanos é aproximadamente $37^{\circ} \mathrm{C}$. (Scott K. Powers., Edward T. Howley).

0 estímulo aos centros termorreguladores hipotalâmicos é oriundo dos receptores da pele e internos. As alterações da temperatura ambiente são detectadas primeiramente pelos receptores térmicos (tanto o calor quanto o frio) localizados na pela. Esses receptores cutâneos da temperatura transmitem impulsos nervosos ao hipotálamo, o qual, então, inicia a resposta adequada num esforço para manter a temperatura do ponto de ajuste do corpo. Além disso, os neurônios sensíveis ao calor/frio estão localizados na medula espinhal e no próprio hipotálamo, detectando alterações da temperatura central. (Scott K. Powers., Edward T. Howley).

Um aumento da temperatura central além do ponto de ajuste faz com que o hipotálamo inicie uma série de ações fisiológicas destinadas a aumentar a quantidade da perda de calor. Em primeiro lugar, o hipotálamo estimula as glândulas sudoríparas, acarretando um aumento da perda de calor pela evaporação. Além disso o centro de controle vasomotor inibe o tônus vasoconstritor normal da pele, promovendo o aumento do fluxo sanguíneo cutâneo e, consequentemente, permitindo o aumento da perda de calor. Quando a temperatura central retorna ao normal, o estímulo promove tanto a sudorese quanto a vasodilatação é removido. Este é um exemplo de sistema de controle que utiliza feed back negativo. (Scott K. Powers., Edward T. Howley).

Quando os receptores ao frio são estimulados na pele ou no hipotálamo, o centro termorregulador estabelece um plano de ação para minimizar a perda de calor e aumentar a sua produção. Primeiro, o centro vasomotor provoca uma vasoconstrição dos vasos sanguíneos periféricos, a qual reduz a perda de calor. Segundo, se a temperatura central diminui significativamente, há inicio de tremor involuntário. As respostas adicionais incluem a estimulação do centro pilomotor, promovendo a piloereção (arrepios). Esse reflexo de piloereção é um meio eficaz de aumentar o espaço de isolamento cutâneo nos animais peludos, mas não é eficiente 
na prevenção de perda de calor nos seres humanos. Além disso, o hipotálamo aumenta indiretamente a produção e a liberação de toxinas, a qual aumenta a produção celular de calor. Finalmente, o hipotálamo posterior inicia a liberação de noradrenalina, que aumenta a taxa do metabolismo celular (termogênese sem ttremor). (Scott K. Powers., Edward T. Howley).

\section{DISTÚRBIOS DA REGULAÇÃO TÉRMICA}

\section{Adrenalina, noradrenalina e dopamina}

Numa situação normal, os sensores térmicos detectam variações da temperatura corporal central e cutânea que transmitem ao centro integrador o qual através de múltiplas vias eferentes promove respostas que visam a conservação ou a dissipação de calor. Anomalias da função ou danos estruturais a qualquer um destes níveis podem resultar na perda da capacidade de regulação térmica. (Magalhães, Sónia etal.)

\section{Temperatura durante o exercício físico}

0 objetivo da regulação de temperatura é a manutenção de uma temperatura interna constante, e com isso, a prevenção do superaquecimento ou do super-resfriamento.

Se a temperatura central deve permanecer constante, a quantidade de calor perdido deve ser proporcional à quantidade de calor ganho. Além disso se a perda de calor é inferior a sua produção, existe um ganho de calor corporal e, consequentemente a temperatura corporal aumenta. Se a perda for maior que a produção, ocorre uma perda de calor e a temperatura corporal diminui. (Scott K. Powers., Edward T. Howley).

Durante o exercício, a temperatura é regulada por meio de ajustes da quantidade de perda de calor. Uma das funções importantes do sistema circulatório é o sistema é o transporte de calor. 0 sangue é muito eficaz nessa função por possuir alta capacidade de armazenar calor. Quando o corpo tende a perder calor, o fluxo sanguíneo cutâneo aumenta como um meio de prover a perda de calor para o meio ambiente. Em contraste, quando o objetivo da regulação da temperatura é impedir a perda de calor, o sangue é desviado da pele e direcionado para o interior do corpo e fim de evitar a perda adicional de calor. (Scott K. Powers., Edward T. Howley).

É importante indicar que, no interior do corpo, a temperatura varia de modo relevante. Isto é, existe um gradiente entre a temperatura interna (áreas centrais profundas, incluindo o coração, os pulmões e os órgãos abdominais) e a temperatura "superficial" (pele). (Scott K. Powers., Edward T. Howley).Em circunstâncias extremas (isto é, exposição a temperaturas muito baixas), a 
temperatura central pode ser $20^{\circ} \mathrm{C}$ maior do que a superficial. No entanto, esses altos gradientes entre a temperatura central e a superficial são raros e a diferença ideal entre elas é de cerca de $4^{\circ} \mathrm{C}(2,45)$. (Scott K. Powers., Edward T. Howley). Mesmo no interior do organismo, a temperatura entre uma parte do corpo e outra, é importante que seja especificado o local onde a temperatura está sendo mensurada. Consequentemente, o termo temperatura corporal é incorreto e deve ser substituído por termos mais descritivos como temperatura central ou temperatura cutânea, dependendo de qual temperatura estiver sendo discutida. (Scott K. Powers., Edward T. Howley).

\section{Termorregulação durante o exercício}

Durante o exercício num ambiente frio/umidade moderada (isto é, umidade e temperatura ambiente baixas). A produção de calor aumenta durante o exercício em virtude da contração muscular e é diretamente proporcional à intensidade do exercício. 0 sangue venoso que drena o músculo em exercício distribui o excesso de calor pelo centro do corpo. (Scott K. Powers., Edward T. Howley). À medida que a temperatura central aumenta, os sensores térmicos hipotalâmicos detectam o aumento da temperatura sanguínea e o centro de integração térmica hipotalâmico compara esse aumento de temperatura com a temperatura do ponto de ajuste e calcula a diferença entre os dois. A resposta é acionar o sistema nervoso para iniciar a transpiração e aumentar o fluxo sanguíneo cutâneo(Scott K. Powers., Edward T. Howley). Essas ações aumentam a perda de calor corporal e minimizam o aumento da temperatura corporal. Nesse ponto, a temperatura interna atinge um novo nível estável elevado. (Scott K. Powers., Edward T. Howley). Essa nova temperatura central estável não representa uma alteração da temperatura do ponto de ajuste, como ocorre na febre. (Scott K. Powers., Edward T. Howley). Em vez disso os centros termorreguladores tentam fazer com que a temperatura central retorne ao nível de repouso, mas são incapazes de fazê-lo em razão da produção sustentada de calor associada ao exercício. (Scott K. Powers., Edward T. Howley). 0 pequeno papel da convecção e da radiação na perda de calor durante exercícios de carga constante num ambiente moderado, se deve a um gradiente de temperatura constante entre a pele e o ambiente. Em contraste, a evaporação possui o papel mais importante na perda de calor durante o exercício nesse tipo de ambiente.

Durante o exercício de carga constante, o aumento da temperatura central está diretamente relacionado com a intensidade do exercício e independe da temperatura ambiente numa ampla faixa de condição $\left(8-29^{\circ} \mathrm{C}\right.$, com umidade relativa baixa). (Scott K. Powers., Edward T. Howley). A temperatura central aumenta conforme a taxa metabólica aumenta. $O$ fato de ser a intensidade do exercício e não a temperatura ambiente que determina a elevação da temperatura central durante o 
exercício sugere que o método de perda de calor durante o exercício contínuo é modificado segundo as condições ambientais. (Scott K. Powers., Edward T. Howley). À medida que a temperatura ambiente aumenta, a taxa de perda de calor por convecção e por radiação diminui em razão da diminuição do gradiente de temperatura entre a pele e o ambiente. Essa diminuição da perda de calor por convecção e por radiação é acompanhada por um aumento da perda de calor por evaporação e a temperatura central permanece a mesma. (Scott K. Powers., Edward T. Howley).

Como foi mencionado, a produção de calor aumenta proporcionalmente à intensidade do exercício. Além disso a perda de calor por convecção e por radiação não aumenta em função da taxa de trabalho. Isto se deve a um gradiente de temperatura relativamente constante entre a pele e o ambiente. Em contraste, existe um aumento consistente da perda de calor por evaporação com os incrementos da intensidade do exercício. Esta observação reenfatiza o ponto de que a evaporação é o principal meio de perder calor durante o exercício. (Scott K. Powers., Edward T. Howley).

\section{Exercício no calor}

0 exercício contínuo num ambiente quente/úmido representa um desafio particularmente estressante para a manutenção da temperatura corporal normal, e da homeostasia de fluidos. 0 calor e a umidade elevados reduzem a capacidade de o corpo perder calor por radiação/convecção e evaporação. Esta incapacidade de perda de calor durante o exercício num ambiente quente/úmido resulta numa maior temperatura central e numa maior taxa de transpiração (maior perda líquida) em comparação com o mesmo exercício realizado num ambiente moderado. (Scott K. Powers., Edward T. Howley).0 efeito combinado da perda líquida e da temperatura central elevada aumenta os riscos de hipertermia e aumento de lesão pelo calor.

\section{CONCLUSÃO}

Os seres Homeotermos, como o homem, necessitam de normotermia função muito importante do sistema nervoso autônomo. Pois a desregulação da temperatura central, pode acarretar à alterações metabólicas e enzimáticas, que podem levar a morte, os seres humanos e muitos animais passam toda a vida a apenas alguns graus do seu ponto de morte pelo calor e pelo frio.

Então devemos sempre tomar atitudes de prevenção, como vestimentas adequadas para a prática de exercícios físicos e esportes, manter-se sempre bem hidratado, além de conscientizar crianças e jovens sobre o perigo da desregulação da temperatura ideal do nosso corpo.

Profissionais da área da saúde envolvidos com toda e qualquer faixa etária, 
devem priorizar a atividade física com aspectos lúdicos e os de competição, e evitar a prática em temperaturas extremas. Pois exercícios em ambientes muito quentes ou frios geram sobrecarga corporal, pois nessas condições o corpo além de se preocupar com o exercício também terá que se preocupar em regular a perda e o ganho de calor à fim de manter normotermia.

\section{REFERENCIAS BIBLIOGRÁFICAS}

1. Faculdade de med. da Universidade do porto. Serviço de fisiologia. T e r m o r r e g u l a çã o. P o r t o, a n o l e t i vo $2001 / 02$ http://www.uff.br/WebQuest/downloads/Termorreg.pdf

2. N e w to n d a C r u z R o cha. Termorregulaçã o. 2005 . http://www.uff.br/fisiovet/Conteudos/termorregulacao.htm

3. Temperatura corporal.MSc LORENA ALMEIDA DE MELO. Fisiologia humana 9 Temperatura corporal. 2014. http://pt.slideshare.net/herbertsantana22/fisiologiahumana-9-temperatura-corporal

4- Neurociências. Fisiologia da termorregulação normal. Thermoregulation. Revista Neurociências V13 N3 (supl-versão eletrônica) -jul/set, 2005. http://revistaneurociencias.com.br/edicoes/2005/RN\%2013\%20SUPLEMENTO/ Pages\%20from\%20RN\%2013\%20SUPLEMENTO-2.pdf

5- FISIOLOGIA DO EXERCÍCIO. TERMORREGULAÇÃO. Linda Moreira. Cooperativa do Fitness.2009. Bibliografia: WEINECK,Jurgen, Manual de treinamento desportivo. São Paulo: Manole, 1989, BOMPA, Tudor, periodização: teoria e metodologia de t r e i n a m e n t o, 4 a e d i çã o, S ã o $\mathrm{P}$ a u lo: p hor t e, 2002.http://www.cdof.com.br/fisio9.htm.

6- Revista Brasileira de Medicina do Esporte vol.4 no.4 Niterói july/ Aug. 1998 http://dx.doi.org/10.1590/S1517-86921998000400002 SOCIEDADE BRASILEIRA DE MEDICINA DO ESPORTE POSICIONAMENTO OFICIAL. Atividade física e saúde na infância e adolescência. http://www.scielo.br/scielo.php?pid=S1517$86921998000400002 \&$ script=sci_arttext\&tlng=pt. 
7- Apostila do curso de fisiologia 2012, Departamento de fisiologia, IB UnespB otucatu. Prof a Silvia M. Nishida. SENTIDO SOMÁTICO. http://www.ibb.unesp.br/Home/Departamentos/Fisiologia/Neuro/06.somestesia .pdf.

8- 0 corpo Humano. Sensibilidade Térmica. NADI. Silvia M. Nishida. http://www2.ibb.unesp.br/nadi/Museu2_qualidade/Museu2_como_funciona/Mus eu_homem_nervoso/Museu2_homem_nervoso_somestesia/Museu2_homem_nervo so_termicos.htm

9- Anatomia e fisiologia humana. Sistema sensoria, Prof ${ }^{a}$ Ana Luisa Miranda Vilela. http://www.afh.bio.br/sentidos/sentidos10.asp.

10 - Fisiologia sensorial. Prof Lucindo Quintas jr (DFS/UFS) 2011. https://erikalizfarma.file.wordpress.com/2011/11/aula_introducao_fisiologia_sen sorial.pdf.

11-SCOTT K. POWERS, EDWARD T. HOWLEY. Fisiologia do Exercício. Teoria e Aplicação ao Condicionamento e ao Desempenho. 5a edição. Manole.

12-Guyton, AC \& Hall, JE, Tratado de Fisiologia Médica. 11ª edição. 2007.

13-Dee Unglaub Silverthorn. Fisiologia humana. Uma abordagem integrada, 5 edição 2010. Artmed.

14-Junqueira \& Carneiro Histologia Básica. Texto e Atlas 12ª edição. Gen/Guanabara Koogan.

15-Rubem David Azulay. David Rubem azulay. Luna azulay-abulafia. Dermatologia 6 edição revisada e atualizada. Gen/Guanabara Koogan. 